\section{Fragilidade entre idosos e percepção de problemas em indicadores de atributos da atenção primária à saúde: resultados do ELSI-Brasil}

\author{
Frailty in elderly individuals and perception of \\ problems in indicators of attributes of primary \\ healthcare: results of the ELSI-Brasil
}

\author{
Fragilidad entre ancianos y percepción de \\ problemas en indicadores de atributos \\ de la atención primaria en salud: \\ resultados del ELSI-Brasil
}

\section{Resumo}

O presente trabalho objetivou examinar a associação entre a síndrome de fragilidade e a percepção de problemas em indicadores de atributos da atenção primária à saúde (APS) entre idosos brasileiros. É um estudo transversal envolvendo 5.432 participantes, com 60 anos ou mais, da primeira onda do Estudo Longitudinal da Saúde dos Idosos Brasileiros (ELSI-Brasil), realizado entre 2015 e 2016. A fragilidade, variável independente, foi definida com base no marco teórico do fenótipo de fragilidade, e os indicadores de problemas em atributos da APS, variáveis dependentes, foram obtidos baseando-se em perguntas relacionadas ao uso de serviços de saúde. Acesso, longitudinalidade, coordenação, integralidade, orientação familiar e adequação cultural foram os atributos avaliados. Utilizou-se modelos de regressão logística ajustados por fatores predisponentes, facilitadores e de necessidade de uso de serviços de saúde para a análise dos dados. Entre os participantes, 55, 1\% eram do sexo feminino, 57,9\% tinham entre 60 e 69 anos e 51,8\% referiram multimorbidade. Idosos frágeis e pré-frágeis representaram 13,4\% e 54,5\% da amostra, respectivamente. Resultados da análise multivariada mostraram que os idosos frágeis em comparação com os robustos apresentaram mais chances de apontar problemas de acesso $(O R=1,45$; IC95\%: 1,08-1,93), longitudinalidade $(O R$ = 1,54; IC95\%: 1,19-2,00) e integralidade $(O R=1,45 ;$ IC95\%: 1,14-1,85), além de maior número de problemas em atributos da APS $(O R=1,38$; IC95\%: 1,05-1,82, para 5 ou mais). O estudo sugere a ocorrência de iniquidades na assistência prestada pela APS brasileira aos idosos frágeis, particularmente no âmbito dos atributos acesso, longitudinalidade e integralidade.

Envelhecimento; Idoso Fragilizado; Serviços de Saúde para Idosos; Pesquisa sobre Serviços de Saúde; Atenção Primária à Saúde

\author{
Alexandre Moreira de Melo Silva 1 \\ Juliana Vaz de Melo Mambrini 1 \\ Juliana Mara Andrade 1 \\ Fabiola Bof de Andrade 1 \\ Maria Fernanda Lima-Costa 1,2
}

doi: 10.1590/0102-311X00255420

\author{
Correspondência \\ A. M. M. Silva \\ Instituto René Rachou, Fundação Oswaldo Cruz. \\ Av. Augusto de Lima 1715, Belo Horizonte, MG \\ 30190-002, Brasil. \\ alexmmsilva@yahoo.com.br \\ 1 Instituto René Rachou, Fundação Oswaldo Cruz, \\ Belo Horizonte, Brasil. \\ 2 Programa de Pós-graduação em Saúde Pública, Universidade \\ Federal de Minas Gerais, Belo Horizonte,
}




\section{Introdução}

O envelhecimento populacional repercute diretamente no planejamento e na prestação de serviços de saúde. Nesse contexto, a síndrome de fragilidade é uma condição clínica de expressiva relevância 1,2. Pode advir de múltiplas causas e ter contribuintes diversos. Conceitualmente, é entendida como um estado de grande vulnerabilidade que proporciona dificuldades à homeostase após a ocorrência de eventos estressores, o que implica maior risco de desfechos adversos como quedas, delirium, incapacidade e morte 1,2,3. Inicialmente reconhecida com base em parâmetros físicos, mais recentemente busca-se avançar na compreensão da síndrome de fragilidade sob um prisma multidimensional, por meio da incorporação, por instrumentos mais novos, de elementos de ordem psicológica, social e ambiental. Ainda que haja divergências em relação ao método mais apropriado para a identificação da fragilidade, o conceito desta condição clínica é consensual 4. Compreendê-la adequadamente pode permitir melhores estimativas de risco-benefício e facilitar a tomada de decisões clínicas pelos profissionais de saúde, além de proporcionar amplo campo para pesquisadores e poder instrumentalizar gestores em planejamentos de serviços de saúde e intervenções populacionais 5,6. Em alguns países, como a Inglaterra, é normativa da política de saúde a identificação e a gestão clínica do idoso frágil 7 .

A equidade é um dos objetivos fundamentais dos sistemas de saúde. Busca-se minimizar disparidades entre subgrupos populacionais, de maneira que populações vulneráveis não sofram desvantagens sistemáticas em relação ao acesso e à qualidade do cuidado 8 . O envelhecimento é um processo heterogêneo. O conceito de fragilidade facilita esse entendimento e, para além de questões clínicas, permite a análise de iniquidades em saúde em relação à população com 60 anos ou mais 9 . Vários estudos têm demonstrado a relevância desse construto em relação à ocorrência de hospitalização, maior tempo de internação e procura por serviços de urgência, e têm reforçado o papel da atenção primária à saúde (APS) bem estruturada para prover cuidados adequados a idosos frágeis, otimizando recursos em saúde 1,2,10. Esse nível de atenção deve representar a base dos sistemas de saúde, e quando bem organizado e qualificado, é mais efetivo tanto do ponto de vista da prestação do cuidado integral quanto do ponto de vista da sustentabilidade dos sistemas 8,11,12. Contudo, para esse fim, é consenso que os serviços de APS precisam ser avaliados, e é desejável que tal avaliação seja pautada pelo grau de desenvolvimento dos atributos essenciais (acesso, longitudinalidade, integralidade e coordenação do cuidado) e derivados (orientação familiar e comunitária e adequação cultural) 8 .

Poucos estudos sobre a avaliação da implementação dos atributos da APS expressaram a perspectiva da atenção ao idoso, menos ainda da atenção ao idoso frágil. Giguere et al. 13 e Woo et al. 14 discutiram, de forma mais ampla, a necessidade de melhorias administrativas e práticas intersetoriais para o melhor cuidado a essa população no contexto da APS local. No Brasil, os trabalhos de Augusto et al. 15 e Araújo et al. 16, apesar de situarem-se nessa temática, foram de âmbito regional e não contemplaram a heterogeneidade da população idosa representada pela fragilidade. No nosso conhecimento, inexistem estudos utilizando amostra representativa da população brasileira com 60 anos ou mais que avaliem o uso de serviços de saúde por idosos frágeis à luz dos atributos da APS.

O presente trabalho objetivou examinar a associação entre a fragilidade e a percepção de problemas em indicadores de atributos da APS entre idosos brasileiros. Pretendeu-se, dessa forma, contribuir para aumentar a compreensão sobre possíveis iniquidades em relação ao uso de serviços de saúde por idosos frágeis no Brasil, ressaltando o papel deste nível de atenção.

\section{Métodos}

\section{Fonte de dados e amostra}

É um estudo transversal baseado em dados da primeira onda do Estudo Longitudinal da Saúde dos Idosos Brasileiros (ELSI-Brasil), realizado entre 2015 e 2016. O ELSI-Brasil é uma coorte prospectiva, de base domiciliar, delineado para representar a população brasileira não institucionalizada com 50 anos ou mais. A amostra foi baseada em estratos de seleção considerando-se o município como unidade primária, seguido por setor censitário e domicílio. Contou-se com 9.412 participantes, residentes em 
70 municípios das cinco regiões do país. Descrição metodológica mais completa pode ser vista na homepage do ELSI-Brasil (http://elsi.cpqrr.fiocruz.br) e em outra publicação 17.

Por ser a fragilidade mais prevalente entre idosos, para o presente trabalho restringiu-se a amostra aos 5.432 participantes da linha de base do ELSI-Brasil que tinham 60 anos ou mais na ocasião da entrevista.

\section{Variáveis dependentes}

As variáveis dependentes deste estudo corresponderam à percepção de problemas em atributos da APS. Aferiu-se as respostas a questões relacionadas ao acesso (necessidade de consultar com o clínico geral para consultar o especialista, facilidade para conseguir consulta, conseguir consulta médica no prazo de 24 horas, conseguir informações por telefone quando necessita de atendimento em saúde), à longitudinalidade (existência de profissional ou serviço de saúde de referência, atendimento pelo mesmo médico que prestou o atendimento anteriormente), à coordenação do cuidado (médico sabe sobre todos os medicamentos em uso, médico conversa sobre os resultados de consulta com especialista, médico conhece os principais problemas de saúde da pessoa), à integralidade (médico tem capacidade de resolver a maioria dos problemas de saúde, orientações sobre alimentação, atividade física, uso de álcool ou tabaco), à orientação familiar (médico questiona sobre saúde e condições de vida dos familiares) e à adequação cultural (médico explica de forma a propiciar entendimento adequado). Apenas as perguntas sobre a necessidade de consultar com o clínico geral para consultar o especialista e sobre a existência de profissional ou serviço de saúde de referência envolveram respostas "sim" ou "não". Todas as outras questionavam a frequência de ocorrência do respectivo indicador. Todas as respostas foram dicotomizadas, sendo as respostas "sim", "sempre" e "na maioria das vezes" categorizadas como "zero", e as respostas "não", "raramente" e "nunca" categorizadas como "um". As respostas as 13 questões foram somadas a fim de obter-se um escore de problemas em APS que, por sua vez, foi dicotomizado com base na mediana. A percepção de até quatro problemas foi categorizada como "zero" e a de cinco ou mais foi categorizada como "um".

\section{Variáveis independentes}

A variável de exposição foi a fragilidade, definida com base no referencial teórico do fenótipo de fragilidade 3, com a análise valendo-se de cinco dimensões: perda de peso não intencional, fraqueza, velocidade de marcha reduzida, autorrelato de exaustão e baixo nível de atividade física. Essas características foram definidas baseando-se em critérios apresentados em publicações anteriores 18,19. A perda de peso foi caracterizada pelo autorrelato de perda de mais de $3 \mathrm{~kg}$ nos últimos três meses. A fraqueza foi avaliada medindo-se a força de preensão manual com o auxílio de um dinamômetro. $\mathrm{O}$ participante foi orientado a realizar o teste por três vezes consecutivas com o membro superior mais forte, sendo considerado o melhor desempenho. Após a estratificação por sexo e índice de massa corporal, a fraqueza foi definida pela força de preensão manual no quintil inferior ou incapacidade para realizar o teste. A velocidade de marcha foi avaliada pelo teste de caminhada 20 . Foi solicitado que o participante caminhasse, de forma habitual, por três metros e cronometrou-se o tempo. Foi considerado o menor tempo entre duas tentativas. Além da incapacidade para realizar o teste, a baixa velocidade de marcha foi caracterizada pelo quintil mais alto de tempo, após a estratificação por sexo e pela média da altura. A exaustão foi definida com base nas respostas às seguintes perguntas da Escala de Depressão do Center for Epidemiological Studies (CES-D) 21: "Na última semana, com que frequência $o$ (a) $\operatorname{Sr}($ a) sentiu que não conseguiria levar adiante suas coisas (iniciava alguma coisa, mas não conseguia terminar)?" e "Na última semana, com que frequência a realização de suas atividades rotineiras exigiram do(a) $\mathrm{Sr}(\mathrm{a})$ um grande esforço para serem realizadas?". O relato de frequências superiores a 3-4 dias em pelo menos uma das questões foi o marcador da presença de exaustão. A atividade física foi avaliada baseando-se em um escore calculado em equivalentes metabólicos por semana, valendose do Short Form of International Physical Activity Questionaire (IPAQ) 22. O baixo nível de atividade física foi definido pelo quintil inferior de equivalentes metabólicos gastos na última semana, estratificado de acordo com o sexo. Foi considerado frágil o idoso com comprometimento de no mínimo três das 
referidas dimensões; pré-frágil, aqueles com comprometimento em uma ou duas dimensões; e robusto, aqueles que não preencheram quaisquer dos cinco critérios.

A inclusão das covariáveis baseou-se na literatura 10,23 e no modelo comportamental de uso de serviços de saúde de Andersen \& Newman 24. Sexo e idade foram utilizados como fatores predisponentes. Como fatores facilitadores, usou-se a renda domiciliar per capita (em quartis), escolaridade (dicotomizada em "até 4 anos" e "5 anos ou mais"), arranjo domiciliar (residir sozinho ou com mais pessoas), local (urbano ou rural) e região de residência (Norte, Nordeste, Centro-oeste, Sudeste, Sul) e afiliação a plano de saúde privado (sim ou não). A presença de doenças e a limitação para realizar atividades básicas da vida diária (ABVDs) foram tratadas como fatores de necessidade para o uso de serviços de saúde. Foi contemplado o autorrelato (sim ou não) das seguintes doenças e condições clínicas: hipertensão arterial, diabetes mellitus, câncer, depressão, doença pulmonar obstrutiva crônica (DPOC), acidente vascular encefálico (AVE), artrite/reumatismo, osteoporose, cardiopatia (englobando o autorrelato de angina, infarto agudo do miocárdio e insuficiência cardíaca). A limitação para a realização de ABVDs foi definida como qualquer dificuldade para realizar pelo menos uma das seguintes atividades: atravessar um cômodo ou andar de um cômodo para outro, deitar-se ou levantar-se da cama, vestir-se, tomar banho, usar o banheiro ou alimentar-se.

\section{Análise estatística}

Inicialmente, foram analisadas as distribuições de frequências das covariáveis para a amostra total e segundo os estratos de fragilidade. Análises de associação bivariadas entre a variável de exposição e os referidos desfechos foram realizadas utilizando-se o teste de qui-quadrado de Pearson, com correção de Rao-Scott para as amostras complexas. A colinearidade, examinada por meio de fatores de inflação da variância, não foi evidenciada $(\mathrm{VIF}<2)$. Modelos de regressão logística foram usados para avaliar a associação entre os desfechos de interesse e a fragilidade, tendo sido ajustados, simultaneamente, pelos fatores predisponentes, facilitadores e de necessidade para o uso de serviços de saúde. Considerando-se a sua relevância como fator de confusão para a discussão dos resultados, foi realizada, adicionalmente, análise estratificada pela fonte de atenção (Sistema Único de Saúde - SUS - e afiliação a plano privado) a fim de examinar a existência de diferenças nas associações entre atributos da APS e fragilidade entre estes dois sistemas. Todos os resultados dos modelos multivariados foram apresentados em odds ratios (OR) com os seus respectivos intervalos de 95\% de confiança (IC95\%).

As análises foram feitas pelo software Stata (https://www.stata.com/), versão 14.2, considerandose a estrutura complexa da amostra (pesos atribuídos aos indivíduos e parâmetros amostrais).

\section{Considerações éticas}

O ELSI-Brasil foi aprovado pelo Comitê de Ética em Pesquisa do Instituto René Rachou, Fundação Oswaldo Cruz, Minas Gerais (CAAE 34649814.3.0000.5091). Os participantes assinaram Termo de Consentimento Livre e Esclarecido.

\section{Resultados}

Dos 9.412 participantes da linha de base do ELSI-Brasil, 5.432 tinham 60 anos ou mais e foram selecionados para a presente análise. Entre esses, 55,1\% eram do sexo feminino, 57,9\% tinham entre $60 \mathrm{e}$ 69 anos e 62,1\% tinham até 4 anos de escolaridade. A maioria residia em zona urbana (84,1\%), sendo $47,6 \%$ oriundos da Região Sudeste, a mais populosa do país. Pouco mais de um quarto da amostra $(26,7 \%)$ informou afiliação a plano de saúde privado e mais da metade $(51,8 \%)$ relataram a presença de multimorbidade (duas ou mais doenças crônicas).

A maioria dos idosos foi classificada como pré-frágil $(54,5 \%)$ e 32,1\% como robustos. Idosos frágeis corresponderam a $13,4 \%$ da amostra, sendo que esta prevalência aumentou em estratos etários superiores. Além da idade, houve diferença estatisticamente significativa entre os estratos de fragilidade em relação à renda domiciliar per capita, escolaridade, região geográfica de residência, afiliação a plano privado de saúde e prevalências de limitação para a realização de ABVDs e multimorbidade. 
Idosos frágeis tinham menor renda e escolaridade, além de terem plano de saúde privado em menor proporção em comparação aos robustos. Cerca de um terço dos idosos frágeis residia no Nordeste brasileiro, apesar de apenas 23,4\% da amostra serem oriundos desta região. Aproximadamente $45 \%$ dos idosos frágeis relataram alguma dificuldade para realizar ABVDs e mais de 67\% disseram ter pelo menos duas doenças crônicas. Mais detalhes podem ser vistos na Tabela 1.

\section{Tabela 1}

Distribuição dos fatores predisponentes, facilitadores e de necessidades para o uso de serviços de saúde em amostra nacional de adultos com 60 anos ou mais, segundo estratos de fragilidade. Estudo Longitudinal da Saúde dos Idosos Brasileiros (ELSI-Brasil), 2015-2016.

\begin{tabular}{|c|c|c|c|c|c|}
\hline Variáveis & $\begin{array}{c}\text { Total } \\
\% \text { (IC95\%) }\end{array}$ & $\begin{array}{l}\text { Robusto } \\
\% \text { (IC95\%) }\end{array}$ & $\begin{array}{l}\text { Pré-frágil } \\
\%(\text { IC95\%) }\end{array}$ & $\begin{array}{c}\text { Frágil } \\
\%(I C 95 \%)\end{array}$ & Valor de $p$ \\
\hline \multicolumn{6}{|l|}{ Fatores predisponentes } \\
\hline Sexo feminino & $\begin{array}{c}55,1 \\
(52,7-57,5)\end{array}$ & $\begin{array}{c}52,4 \\
(49,0-55,8)\end{array}$ & $\begin{array}{c}55,8 \\
(52,9-58,6)\end{array}$ & $\begin{array}{c}59,0 \\
(53,7-64,2)\end{array}$ & 0,060 \\
\hline Idade (anos) & & & & & $<0,001$ * \\
\hline $60-69$ & $\begin{array}{c}57,9 \\
(55,5-60,3)\end{array}$ & $\begin{array}{c}70,0 \\
(66,7-73,2)\end{array}$ & $\begin{array}{c}56,3 \\
(53,5-59,0)\end{array}$ & $\begin{array}{c}35,6 \\
(30,7-40,8)\end{array}$ & \\
\hline $70-79$ & $\begin{array}{c}29,7 \\
(27,8-31,7)\end{array}$ & $\begin{array}{c}25,6 \\
(22,9-28,5)\end{array}$ & $\begin{array}{c}30,9 \\
(28,3-33,7)\end{array}$ & $\begin{array}{c}34,9 \\
(30,9-39,0)\end{array}$ & \\
\hline 80 ou mais & $\begin{array}{c}12,4 \\
(10,7-14,3)\end{array}$ & $\begin{array}{c}4,4 \\
(3,4-5,7)\end{array}$ & $\begin{array}{c}12,8 \\
(10,9-15,0)\end{array}$ & $\begin{array}{c}29,6 \\
(25,3-34,3)\end{array}$ & \\
\hline \multicolumn{6}{|l|}{ Fatores facilitadores } \\
\hline Renda domiciliar mensal per capita (quartil inferior) & $\begin{array}{c}19,3 \\
(16,7-22,1)\end{array}$ & $\begin{array}{c}14,8 \\
(12,3-17,8)\end{array}$ & $\begin{array}{c}20,2 \\
(17,5-23,2)\end{array}$ & $\begin{array}{c}26,0 \\
(20,9-31,9)\end{array}$ & $<0,001$ * \\
\hline Escolaridade (até 4 anos) & $\begin{array}{c}62,1 \\
(58,6-65,4)\end{array}$ & $\begin{array}{c}52,0 \\
(47,4-56,5)\end{array}$ & $\begin{array}{c}64,7 \\
(60,8-68,4)\end{array}$ & $\begin{array}{c}75,8 \\
(71,0-80,1)\end{array}$ & $<0,001$ * \\
\hline Região de residência & & & & & 0,001 * \\
\hline Sudeste & $\begin{array}{c}47,6 \\
(36,0-59,4)\end{array}$ & $\begin{array}{c}49,7 \\
(37,9-61,5)\end{array}$ & $\begin{array}{c}47,7 \\
(36,2-59,5)\end{array}$ & $\begin{array}{c}41,7 \\
(28,4-56,4)\end{array}$ & \\
\hline Nordeste & $\begin{array}{c}23,4 \\
(15,2-34,4)\end{array}$ & $\begin{array}{c}17,8 \\
(11,0-27,4)\end{array}$ & $\begin{array}{c}24,4 \\
(15,9-35,6)\end{array}$ & $\begin{array}{c}32,9 \\
(20,5-48,3)\end{array}$ & \\
\hline Sul & $\begin{array}{c}17,3 \\
(9,0-30,6)\end{array}$ & $\begin{array}{c}19,8 \\
(10,7-33,7)\end{array}$ & $\begin{array}{c}16,5 \\
(8,5-29,5)\end{array}$ & $\begin{array}{c}14,4 \\
(5,9-30,9)\end{array}$ & \\
\hline Centro-oeste & $\begin{array}{c}6,3 \\
(2,9-13,2)\end{array}$ & $\begin{array}{c}7,6 \\
(3,4-16,0)\end{array}$ & $\begin{array}{c}5,8 \\
(2,6-12,2)\end{array}$ & $\begin{array}{c}5,3 \\
(2,4-11,5)\end{array}$ & \\
\hline Norte & $\begin{array}{c}5,4 \\
(2,2-12,7)\end{array}$ & $\begin{array}{c}5,2 \\
(2,2-11,5)\end{array}$ & $\begin{array}{c}5,6 \\
(2,1-13,7)\end{array}$ & $\begin{array}{c}5,7 \\
(2,3-13,0)\end{array}$ & \\
\hline Residência em zona urbana & $\begin{array}{c}84,1 \\
(78,3-88,6)\end{array}$ & $\begin{array}{c}84,1 \\
(77,8-88,9)\end{array}$ & $\begin{array}{c}84,1 \\
(78,2-88,6)\end{array}$ & $\begin{array}{c}84,1 \\
(76,4-89,7)\end{array}$ & 0,999 \\
\hline Mora sozinho & $\begin{array}{c}11,9 \\
(10,7-13,1)\end{array}$ & $\begin{array}{c}11,3 \\
(10,0-12,6)\end{array}$ & $\begin{array}{c}12,5 \\
(11,0-14,2)\end{array}$ & $\begin{array}{c}10,8 \\
(8,6-13,4)\end{array}$ & 0,224 \\
\hline Afiliação a plano privado & $\begin{array}{c}26,7 \\
(23,7-30,0)\end{array}$ & $\begin{array}{c}31,0 \\
(27,2-35,1)\end{array}$ & $\begin{array}{c}24,7 \\
(21,6-28,2)\end{array}$ & $\begin{array}{c}24,7 \\
(19,0-31,5)\end{array}$ & 0,009 * \\
\hline \multicolumn{6}{|l|}{ Fatores de necessidade } \\
\hline Limitação para realizar ABVDs ** & $\begin{array}{c}16,6 \\
(14,9-18,5)\end{array}$ & $\begin{array}{c}5,0 \\
(3,6-6,8)\end{array}$ & $\begin{array}{c}16,6 \\
(14,4-19,0)\end{array}$ & $\begin{array}{c}44,9 \\
(39,1-50,7)\end{array}$ & $<0,001$ * \\
\hline Duas ou mais doenças crônicas *** & $\begin{array}{c}51,8 \\
(49,4-54,3)\end{array}$ & $\begin{array}{c}41,3 \\
(38,3-44,3)\end{array}$ & $\begin{array}{c}54,2 \\
(50,8-57,7)\end{array}$ & $\begin{array}{c}67,4 \\
(62,8-71,7)\end{array}$ & $<0,001$ * \\
\hline
\end{tabular}

ABVD: atividades básicas da vida diária; IC95\%: intervalo de 95\% de confiança.

Nota: os valores de proporção (\%) e seus respectivos IC95\% consideram o delineamento da amostra e os pesos individuais.

* $p<0,05$. Teste qui-quadrado de Pearson com correção de Rao-Scott para diferença entre os grupos;

** Dificuldade para realizar pelo menos uma das seguintes atividades: atravessar um cômodo ou andar de um cômodo para outro, deitar-se ou levantar-se da cama, vestir-se, tomar banho, usar o banheiro e alimentar-se;

** Hipertensão arterial, diabetes mellitus, câncer, depressão, doença pulmonar obstrutiva crônica (DPOC), acidente vascular encefálico (AVE), artrite/ reumatismo, osteoporose, cardiopatia (angina, infarto agudo do miocárdio, insuficiência cardíaca). 
A Tabela 2 apresenta a associação entre os estratos de fragilidade e problemas em indicadores de atributos da APS. De forma geral, a prevalência de tais problemas variou entre 14,4\% (médico não sabe sobre todos os medicamentos que está tomando - coordenação do cuidado) e 71,1\% (médico não pergunta sobre a saúde e condições de vida dos familiares - orientação familiar). Houve diferença estatisticamente significativa entre os estratos de fragilidade em relação ao relato de dificuldade para conseguir uma consulta (acesso), à percepção de que o médico assistente não era capaz de resolver a maioria dos problemas de saúde (integralidade) e à presença de cinco ou mais problemas em indicadores de atributos da APS. A percepção desses problemas foi significativamente maior entre os idosos frágeis em comparação aos robustos.

A Tabela 3 mostra os resultados da análise multivariada entre os estratos de fragilidade e os problemas em indicadores dos atributos da APS. Não houve diferença estatisticamente significativa em relação ao relato de tais problemas entre os idosos robustos e pré-frágeis. Entretanto, os idosos frágeis, quando comparados aos robustos, apresentaram mais chances de relatar dificuldade para conseguir consulta (OR = 1,45; IC95\%: 1,08-1,93), não ser atendido pelo mesmo médico (OR = 1,54; IC95\%:

Tabela 2

Associação entre os estratos de fragilidade e problemas em indicadores dos atributos da atenção primária à saúde (APS) em amostra nacional de adultos com 60 anos ou mais. Estudo Longitudinal da Saúde dos Idosos Brasileiros (ELSI-Brasil), 2015-2016.

\begin{tabular}{|c|c|c|c|c|c|}
\hline Problemas em atributos da APS & $\begin{array}{l}\text { Total } \\
\%(\text { IC95\%) }\end{array}$ & $\begin{array}{l}\text { Robusto } \\
\%(\text { IC95\%) }\end{array}$ & $\begin{array}{l}\text { Pré-frágil } \\
\%(\text { IC95\%) }\end{array}$ & $\begin{array}{l}\text { Frágil } \\
\%(I C 95 \%)\end{array}$ & Valor de $p$ \\
\hline $\begin{array}{l}\text { Não precisa realizar consulta com o clínico geral para consultar } \\
\text { o especialista (acesso) }\end{array}$ & $\begin{array}{c}28,1 \\
(25,6-30,9)\end{array}$ & $\begin{array}{c}30,8 \\
(27,3-34,4)\end{array}$ & $\begin{array}{c}26,6 \\
(23,7-29,8)\end{array}$ & $\begin{array}{c}28,1 \\
(22,9-33,9)\end{array}$ & 0,114 \\
\hline Não acha fácil conseguir uma consulta (acesso) & $\begin{array}{c}35,6 \\
(32,7-38,6)\end{array}$ & $\begin{array}{c}31,8 \\
(27,8-36,0)\end{array}$ & $\begin{array}{c}36,1 \\
(33,1-39,2)\end{array}$ & $\begin{array}{c}42,5 \\
(37,6-47,6)\end{array}$ & $<0,001 *$ \\
\hline Não consegue consulta em 24 horas (acesso) & $\begin{array}{c}47,3 \\
(43,6-51,0)\end{array}$ & $\begin{array}{c}46,3 \\
(41,5-51,2)\end{array}$ & $\begin{array}{c}47,0 \\
(43,1-50,8)\end{array}$ & $\begin{array}{c}50,9 \\
(46,2-55,7)\end{array}$ & 0,199 \\
\hline Não consegue pedir informações por telefone (acesso) & $\begin{array}{c}58,6 \\
(55,1-62,0)\end{array}$ & $\begin{array}{c}58,7 \\
(54,3-63,0)\end{array}$ & $\begin{array}{c}58,2 \\
(54,0-62,2)\end{array}$ & $\begin{array}{c}59,9 \\
(54,6-64,9)\end{array}$ & 0,845 \\
\hline $\begin{array}{l}\text { Não tem profissional ou serviço de saúde de referência } \\
\text { (longitudinalidade) }\end{array}$ & $\begin{array}{c}35,2 \\
(32,1-38,3)\end{array}$ & $\begin{array}{c}36,8 \\
(32,0-41,8)\end{array}$ & $\begin{array}{c}34,7 \\
(32,0-37,6)\end{array}$ & $\begin{array}{c}33,0 \\
(28,8-37,6)\end{array}$ & 0,306 \\
\hline Não é atendido pelo mesmo médico (longitudinalidade) & $\begin{array}{c}33,1 \\
(30,4-35,8)\end{array}$ & $\begin{array}{c}31,0 \\
(27,7-34,6)\end{array}$ & $\begin{array}{c}33,3 \\
(30,4-36,3)\end{array}$ & $\begin{array}{c}37,1 \\
(32,2-42,2)\end{array}$ & 0,067 \\
\hline $\begin{array}{l}\text { Médico não sabe sobre todos os medicamentos que está } \\
\text { tomando (coordenação) }\end{array}$ & $\begin{array}{c}14,4 \\
(12,7-16,3)\end{array}$ & $\begin{array}{c}15,4 \\
(12,8-18,3)\end{array}$ & $\begin{array}{c}13,8 \\
(11,8-16,2)\end{array}$ & $\begin{array}{c}14,8 \\
(12,0-18,1)\end{array}$ & 0,525 \\
\hline $\begin{array}{l}\text { Médico não conversa sobre os resultados da consulta com o } \\
\text { especialista (coordenação) }\end{array}$ & $\begin{array}{c}46,2 \\
(43,8-48,6)\end{array}$ & $\begin{array}{c}44,0 \\
(40,1-47,9)\end{array}$ & $\begin{array}{c}46,5 \\
(43,9-49,1)\end{array}$ & $\begin{array}{c}50,6 \\
(43,9-57,2)\end{array}$ & 0,158 \\
\hline $\begin{array}{l}\text { Médico não sabe quais são seus principais problemas de saúde } \\
\text { (coordenação) }\end{array}$ & $\begin{array}{c}16,6 \\
(14,8-18,6)\end{array}$ & $\begin{array}{c}16,2 \\
(13,9-18,8)\end{array}$ & $\begin{array}{c}16,4 \\
(14,0-19,0)\end{array}$ & $\begin{array}{c}18,7 \\
(14,6-23,5)\end{array}$ & 0,546 \\
\hline $\begin{array}{l}\text { Médico não é capaz de resolver a maioria dos problemas de } \\
\text { saúde (integralidade) }\end{array}$ & $\begin{array}{c}22,3 \\
(20,5-24,1)\end{array}$ & $\begin{array}{c}19,9 \\
(17,5-22,5)\end{array}$ & $\begin{array}{c}22,4 \\
(20,5-24,5)\end{array}$ & $\begin{array}{c}27,1 \\
(23,4-31,1)\end{array}$ & $0,001 *$ \\
\hline $\begin{array}{l}\text { Médico não conversa sobre cuidados com alimentação, } \\
\text { atividade física, uso de álcool ou tabaco (integralidade) }\end{array}$ & $\begin{array}{c}30,7 \\
(28,6-33,0)\end{array}$ & $\begin{array}{c}30,0 \\
(27,1-33,1)\end{array}$ & $\begin{array}{c}31,3 \\
(29,0-33,7)\end{array}$ & $\begin{array}{c}30,0 \\
(25,5-34,9)\end{array}$ & 0,671 \\
\hline $\begin{array}{l}\text { Médico não pergunta sobre a saúde e condições de vida dos } \\
\text { familiares (orientação familiar) }\end{array}$ & $\begin{array}{c}71,1 \\
(68,9-73,2)\end{array}$ & $\begin{array}{c}70,8 \\
(67,7-73,6)\end{array}$ & $\begin{array}{c}70,8 \\
(68,0-73,5)\end{array}$ & $\begin{array}{c}73,3 \\
(67,8-78,1)\end{array}$ & 0,608 \\
\hline $\begin{array}{l}\text { Médico não explica de forma que possa entender (adequação } \\
\text { cultural) }\end{array}$ & $\begin{array}{c}17,5 \\
(15,8-19,4)\end{array}$ & $\begin{array}{c}16,5 \\
(14,0-19,4)\end{array}$ & $\begin{array}{c}17,3 \\
(15,5-19,2)\end{array}$ & $\begin{array}{c}21,0 \\
(17,3-25,3)\end{array}$ & 0,070 \\
\hline Relato de 5 ou mais problemas em atributos da APS ** & $\begin{array}{c}43,2 \\
(40,1-46,3)\end{array}$ & $\begin{array}{c}39,6 \\
(34,9-44,6)\end{array}$ & $\begin{array}{c}44,3 \\
(40,9-47,7)\end{array}$ & $\begin{array}{c}47,1 \\
(41,8-52,4)\end{array}$ & 0,043 * \\
\hline
\end{tabular}

IC95\%: intervalo de 95\% de confiança.

Nota: os valores de proporção (\%) e seus respectivos IC95\% consideram o delineamento da amostra e os pesos individuais.

* $p<0,05$. Teste qui-quadrado de Pearson com correção de Rao-Scott para diferença entre os grupos;

** Dicotomizado com base na mediana (mediana $=4$ ). 
Tabela 3

Resultados da análise multivariada entre os estratos de fragilidade e problemas em indicadores dos atributos da atenção primária à saúde (APS) em amostra nacional de adultos com 60 anos ou mais, estratificada pela fonte de atenção em saúde. Estudo Longitudinal da Saúde dos Idosos Brasileiros (ELSI-Brasil), 2015-2016.

\begin{tabular}{|c|c|c|c|c|c|c|}
\hline \multirow[t]{2}{*}{ Problemas em atributos da APS } & \multicolumn{2}{|c|}{ Amostra total } & \multicolumn{2}{|c|}{ SUS } & \multicolumn{2}{|c|}{ Plano privado } \\
\hline & $\begin{array}{l}\text { Pré-frágil } \\
\text { OR (IC95\%) }\end{array}$ & $\begin{array}{l}\text { Frágil } \\
\text { OR (IC95\%) }\end{array}$ & $\begin{array}{l}\text { Pré-frágil } \\
\text { OR (IC95\%) }\end{array}$ & $\begin{array}{l}\text { Frágil } \\
\text { OR (IC95\%) }\end{array}$ & $\begin{array}{l}\text { Pré-frágil } \\
\text { OR (IC95\%) }\end{array}$ & $\begin{array}{c}\text { Frágil } \\
\text { OR (IC95\%) }\end{array}$ \\
\hline $\begin{array}{l}\text { Não precisa realizar consulta com o clínico geral } \\
\text { para consultar o especialista (acesso) }\end{array}$ & $\begin{array}{c}0,84 \\
(0,69-1,03)\end{array}$ & $\begin{array}{c}0,92 \\
(0,65-1,31)\end{array}$ & $\begin{array}{c}0,82 \\
(0,64-1,06)\end{array}$ & $\begin{array}{c}0,79 \\
(0,54-1,15)\end{array}$ & $\begin{array}{c}0,87 \\
(0,62-1,21)\end{array}$ & $\begin{array}{c}1,41 \\
(0,75-2,67)\end{array}$ \\
\hline Não acha fácil conseguir uma consulta (acesso) & $\begin{array}{c}1,12 \\
(0,96-1,31)\end{array}$ & $\begin{array}{c}1,45 * \\
(1,08-1,93)\end{array}$ & $\begin{array}{c}1,19 \\
(1,0-1,42)\end{array}$ & $\begin{array}{c}1,54 \text { * } \\
(1,14-2,09)\end{array}$ & $\begin{array}{c}0,94 \\
(0,70-1,27)\end{array}$ & $\begin{array}{c}1,24 \\
(0,66-2,34)\end{array}$ \\
\hline Não consegue consulta em 24 horas (acesso) & $\begin{array}{c}1,02 \\
(0,86-1,21)\end{array}$ & $\begin{array}{c}1,22 \\
(0,98-1,52)\end{array}$ & $\begin{array}{c}1,11 \\
(0,91-1,36)\end{array}$ & $\begin{array}{c}1,45 * \\
(1,14-1,86)\end{array}$ & $\begin{array}{c}0,82 \\
(0,56-1,20)\end{array}$ & $\begin{array}{c}0,74 \\
(0,47-1,17)\end{array}$ \\
\hline $\begin{array}{l}\text { Não consegue pedir informações por telefone } \\
\text { (acesso) }\end{array}$ & $\begin{array}{c}0,94 \\
(0,79-1,12)\end{array}$ & $\begin{array}{c}1,03 \\
(0,77-1,37)\end{array}$ & $\begin{array}{c}0,84 \\
(0,69-1,04)\end{array}$ & $\begin{array}{c}0,88 \\
(0,62-1,24)\end{array}$ & $\begin{array}{c}1,19 \\
(0,86-1,64)\end{array}$ & $\begin{array}{c}1,51 \\
(0,89-2,57)\end{array}$ \\
\hline $\begin{array}{l}\text { Não tem profissional ou serviço de saúde de } \\
\text { referência (longitudinalidade) }\end{array}$ & $\begin{array}{c}1,00 \\
(0,82-1,22)\end{array}$ & $\begin{array}{c}1,09 \\
(0,81-1,45)\end{array}$ & $\begin{array}{c}1,08 \\
(0,86-1,34)\end{array}$ & $\begin{array}{c}1,18 \\
(0,81-1,71)\end{array}$ & $\begin{array}{c}0,83 \\
(0,58-1,19)\end{array}$ & $\begin{array}{c}0,85 \\
(0,48-1,50)\end{array}$ \\
\hline $\begin{array}{l}\text { Não é atendido pelo mesmo médico } \\
\text { (longitudinalidade) }\end{array}$ & $\begin{array}{c}1,17 \\
(0,99-1,37)\end{array}$ & $\begin{array}{c}1,54 * \\
(1,19-2,00)\end{array}$ & $\begin{array}{c}1,23 \\
(0,99-1,52)\end{array}$ & $\begin{array}{c}1,69 * \\
(1,25-2,27)\end{array}$ & $\begin{array}{c}1,01 \\
(0,66-1,54)\end{array}$ & $\begin{array}{c}1,16 \\
(0,66-2,05)\end{array}$ \\
\hline $\begin{array}{l}\text { Médico não sabe sobre todos os medicamentos } \\
\text { que está tomando (coordenação) }\end{array}$ & $\begin{array}{c}0,92 \\
(0,73-1,16)\end{array}$ & $\begin{array}{c}1,01 \\
(0,72-1,43)\end{array}$ & $\begin{array}{c}0,92 \\
(0,72-1,18)\end{array}$ & $\begin{array}{c}1,09 \\
(0,74-1,60)\end{array}$ & $\begin{array}{c}0,91 \\
(0,54-1,51)\end{array}$ & $\begin{array}{c}0,65 \\
(0,30-1,41)\end{array}$ \\
\hline $\begin{array}{l}\text { Médico não conversa sobre os resultados da } \\
\text { consulta com o especialista (coordenação) }\end{array}$ & $\begin{array}{c}1,11 \\
(0,93-1,33)\end{array}$ & $\begin{array}{c}1,36 \\
(1,00-1,85)\end{array}$ & $\begin{array}{c}1,19 \\
(0,96-1,48)\end{array}$ & $\begin{array}{c}1,43 \\
(0,97-2,11)\end{array}$ & $\begin{array}{c}0,93 \\
(0,68-1,26)\end{array}$ & $\begin{array}{c}1,21 \\
(0,71-2,06)\end{array}$ \\
\hline $\begin{array}{l}\text { Médico não sabe quais são seus principais } \\
\text { problemas de saúde (coordenação) }\end{array}$ & $\begin{array}{c}1,04 \\
(0,83-1,29)\end{array}$ & $\begin{array}{c}1,29 \\
(0,90-1,84)\end{array}$ & $\begin{array}{c}1,08 \\
(0,82-1,41)\end{array}$ & $\begin{array}{c}1,43 \\
(0,96-2,12)\end{array}$ & $\begin{array}{c}0,91 \\
(0,57-1,43)\end{array}$ & $\begin{array}{c}0,72 \\
(0,18-2,94)\end{array}$ \\
\hline $\begin{array}{l}\text { Médico não é capaz de resolver a maioria dos } \\
\text { problemas de saúde (integralidade) }\end{array}$ & $\begin{array}{c}1,14 \\
(0,96-1,34)\end{array}$ & $\begin{array}{c}1,45 * \\
(1,14-1,85)\end{array}$ & $\begin{array}{c}0,99 \\
(0,82-1,21)\end{array}$ & $\begin{array}{c}1,36 \text { * } \\
(1,03-1,81)\end{array}$ & $\begin{array}{c}2,01 * \\
(1,38-2,95)\end{array}$ & $\begin{array}{c}1,91 * \\
(1,04-3,52)\end{array}$ \\
\hline $\begin{array}{l}\text { Médico não conversa sobre cuidados com } \\
\text { alimentação, atividade física, uso de álcool ou } \\
\text { tabaco (integralidade) }\end{array}$ & $\begin{array}{c}1,11 \\
(0,92-1,33)\end{array}$ & $\begin{array}{c}1,06 \\
(0,79-1,41)\end{array}$ & $\begin{array}{c}1,05 \\
(0,87-1,28)\end{array}$ & $\begin{array}{c}0,92 \\
(0,68-1,23)\end{array}$ & $\begin{array}{c}1,34 \\
(0,87-2,05)\end{array}$ & $\begin{array}{c}1,92 * \\
(1,02-3,62)\end{array}$ \\
\hline $\begin{array}{l}\text { Médico não pergunta sobre a saúde e condições } \\
\text { de vida dos familiares (orientação familiar) }\end{array}$ & $\begin{array}{c}0,98 \\
(0,82-1,17)\end{array}$ & $\begin{array}{c}1,11 \\
(0,84-1,47)\end{array}$ & $\begin{array}{c}1,05 \\
(0,86-1,28)\end{array}$ & $\begin{array}{c}1,29 \\
(0,95-1,75)\end{array}$ & $\begin{array}{c}0,84 \\
(0,59-1,21)\end{array}$ & $\begin{array}{c}0,79 \\
(0,41-1,50)\end{array}$ \\
\hline $\begin{array}{l}\text { Médico não explica de forma que possa } \\
\text { entender (adequação cultural) }\end{array}$ & $\begin{array}{c}1,00 \\
(0,83-1,20)\end{array}$ & $\begin{array}{c}1,31 \\
(0,97-1,77)\end{array}$ & $\begin{array}{c}0,98 \\
(0,80-1,21)\end{array}$ & $\begin{array}{c}1,33 \\
(0,94-1,89)\end{array}$ & $\begin{array}{c}1,09 \\
(0,70-1,70)\end{array}$ & $\begin{array}{c}1,24 \\
(0,51-3,01)\end{array}$ \\
\hline $\begin{array}{l}\text { Relato de } 5 \text { ou mais problemas em atributos da } \\
\text { APS ** }\end{array}$ & $\begin{array}{c}1,18 \\
(0,96-1,45)\end{array}$ & $\begin{array}{c}1,38 * \\
(1,05-1,82)\end{array}$ & $\begin{array}{c}1,21 \\
(0,97-1,49)\end{array}$ & $\begin{array}{c}1,41 * \\
(1,05-1,91)\end{array}$ & $\begin{array}{c}1,12 \\
(0,73-1,71)\end{array}$ & $\begin{array}{c}1,27 \\
(0,69-2,33)\end{array}$ \\
\hline
\end{tabular}

IC95\%: intervalo de 95\% de confiança; OR: odds ratio; SUS: Sistema Único de Saúde.

Nota: categoria de referência - idoso robusto. Modelos de regressão logística ajustados por sexo, idade, escolaridade, renda domiciliar per capita, arranjo domiciliar, local e região de residência, afiliação a plano de saúde (amostra total), comorbidades e limitação para realizar atividades básicas da vida diária.

* $\mathrm{p}<0,05$;

** Dicotomizado com base na mediana (mediana $=4)$.

1,19-2,00) e de considerar que o médico assistente não era capaz de resolver a maioria dos problemas de saúde (OR = 1,45; IC95\%: 1,14-1,85), além de maior chance de relatar 5 ou mais problemas em indicadores dos atributos da APS (OR = 1,38; IC95\%: 1,05-1,82). Ao estratificar as análises pela fonte de atenção à saúde, os resultados entre os usuários do SUS foram semelhantes àqueles referentes à amostra total. Os mesmos problemas apresentaram mais chances de terem sido reportados por idosos frágeis em relação aos robustos, sendo as medidas de efeito semelhantes (OR = 1,54; IC95\%: 1,14-2,09, para dificuldade para conseguir consulta; OR = 1,69; IC95\%: 1,25-2,27, para não ser atendido pelo mesmo médico; OR = 1,36; IC95\%: 1,03-1,81, para o relato de que o médico assistente não era capaz 
de resolver a maioria dos problemas de saúde e; OR =1,41; IC95\%: 1,05-1,91, para o relato de 5 ou mais problemas em indicadores dos atributos da APS). Já entre os usuários de plano de saúde privado, tanto os idosos frágeis quanto os pré-frágeis, em comparação aos robustos, registraram mais chances de relatar que o médico não era capaz de resolver a maioria dos problemas de saúde $(\mathrm{OR}=1,91$; IC95\%: 1,04-3,52 e OR = 2,01; IC95\%: 1,38-2,95, respectivamente). Além disso, não conversar sobre aspectos de prevenção e promoção à saúde também apresentou associação com a fragilidade. Idosos frágeis apresentaram maior chance de relatar essa percepção quando comparados aos idosos robustos $(\mathrm{OR}=1,92$; IC95\%: 1,02-3,62). Nesse quesito, não houve diferença entre o grupo de referência e os idosos pré-frágeis. Análises de interação entre as variáveis fragilidade e plano de saúde (não mostradas) apontaram resultados na mesma direção, ou seja, a afiliação a plano de saúde privado modificou o efeito da fragilidade em relação à resolutividade médica e ao relato da falta de diálogo sobre hábitos de vida, como cuidados com a alimentação, atividade física, uso de álcool e tabagismo.

\section{Discussão}

Os resultados apresentados sugerem que idosos brasileiros frágeis, quando comparados a idosos robustos, encontram mais problemas quando demandam cuidados em APS, independentemente de fatores predisponentes, facilitadores e de necessidade. Apesar da evidente maior complexidade clínica dos idosos frágeis e do maior uso de serviços de saúde 3,10,25, essa condição, altamente vulnerável, foi associada a maiores problemas de acesso, longitudinalidade e integralidade na atenção à saúde. Não foram evidenciadas, por outro lado, diferenças estatisticamente significantes nas questões acerca da coordenação do cuidado, orientação familiar e adequação cultural. Quando examinados separadamente por fonte de atenção à saúde, os idosos frágeis usuários do SUS encontraram mais problemas em relação ao acesso, longitudinalidade e integralidade, ao passo que entre afiliados a planos privados a percepção de problemas relacionou-se basicamente à integralidade.

Neste estudo, o instrumento usado para a definição dos estratos de fragilidade foi construído com base no fenótipo de fragilidade 3, construto validado, reconhecido e que, apesar de apresentar várias formas de aferição, é um dos mais utilizados em estudos epidemiológicos 1,2,25,26. Usando esse conceito, os resultados deste trabalho mostram que o idoso frágil brasileiro, em comparação a idosos robustos, é mais velho, tem menor renda e escolaridade e é portador de mais doenças crônicas e incapacidades. Esse perfil, mais detalhado em outro estudo 19, também foi observado em outros países 3,25.

O rastreamento da fragilidade entre idosos tende ao consenso na literatura 2,7,10,27, estando, vários instrumentos, disponíveis 4,27. Por ser uma condição potencialmente dinâmica, intervenções precoces em fatores de risco ou determinantes de um estado pré-frágil podem evitar ou atrasar a progressão para a fragilidade 1,2,27. Dados do estudo SHARE, envolvendo dez países europeus, mostraram que os idosos frágeis aumentam a utilização da APS e de serviços hospitalares antes do início da incapacidade 10. Nesse sentido, é fundamental que o sistema de saúde opere no sentido de evitar ou retardar o processo de fragilização, além de proporcionar cuidado adequado aos idosos frágeis, atendendo às necessidades com respeito às preferências e valores 2. Para tanto, seria oportuno que a APS brasileira, tal qual a APS inglesa 7, rastreasse os idosos adscritos nessa perspectiva. Instrumentos adequados para uso em APS devem ser acurados, curtos e de fácil aplicação por profissionais não especialistas 4 . O índice de vulnerabilidade clínico-funcional 20 (IVCF-20) é uma das alternativas 4,28, podendo ser aplicado por agentes comunitários treinados. Uma vez identificados, os idosos frágeis e em risco de fragilização (pré-frágeis) seriam submetidos à avaliação multidimensional ainda na APS. Condições mais complexas demandariam a realização de avaliação geriátrica ampla e o compartilhamento do cuidado com equipe multiprofissional e outros especialistas na atenção secundária. Nessa ótica, a estruturação objetiva do trabalho em APS é uma das possibilidades de se ampliar o acesso e coordenar, de forma integral, o cuidado ao longo do tempo.

Sistemas de saúde embasados em APS têm melhores resultados em relação àqueles que não se estruturam valendo-se deste nível de atenção 8,11,12. Segundo Starfield 8, a APS pode ser mais bem avaliada pelo nível de desenvolvimento de seus atributos. Esses, por sua vez, se entrelaçam e são interdependentes. Barreiras de acesso limitam a longitudinalidade, integralidade e coordenação ${ }^{8}$. Em geral, sistemas de saúde desenhados para diagnóstico e tratamento de condições agudas não acolhem 
adequadamente pessoas com condições multidimensionais complexas associadas a fatores familiares, dos cuidadores e ambientais 6,13. Questões inerentes à complexidade clínica e contextual do idoso frágil brasileiro e à forma de organização dos serviços de saúde 6 podem ajudar na compreensão dos resultados apresentados, os quais sugerem que esta população pode encontrar mais problemas para conseguir consulta médica e alcançar cuidados longitudinais e integrais satisfatórios. Partindo-se da percepção de que mesmo entre diferentes países a maioria dos problemas relacionados à APS são estruturais 29 , proceder a adequações administrativas e intersetoriais mais centradas nos usuários pode ser pertinente e contribuir para a efetividade da atenção à saúde 12,13,14. Nesse sentido, a assistência domiciliar oportuna, braço importante da APS, reconhecida promotora da equidade do cuidado em saúde a populações vulneráveis 30 , pode ser a única alternativa para o cuidado de alguns subgrupos de idosos frágeis. Para esses, as limitações clínicas e de mobilidade configuram barreiras de acesso, o que pode repercutir no desenvolvimento dos outros atributos e na maior procura por serviços de urgência 13 , aumentando as probabilidades de insucesso terapêutico e iatrogenias. Acesso em tempo oportuno, cuidado equitativo e centrado na pessoa representam alguns dos objetivos estruturais da APS e dos sistemas de saúde em geral 12. No Brasil, nas últimas décadas, a expansão da Estratégia Saúde da Família (ESF) foi responsável por melhorias no acesso, longitudinalidade e utilização de serviços de saúde por idosos, pessoas com menor renda e portadores de doenças 11. Intensificar a implementação das normativas das políticas de saúde que tratam da maior qualificação das equipes de APS na perspectiva do cuidado gerontológico 31 e otimizar os sistemas de referência e contrarreferência, presencial ou à distância, contribuirão ainda mais para o desenvolvimento dos atributos de longitudinalidade e integralidade e, consequentemente, para a melhora da performance da APS $12 \mathrm{em}$ relação à assistência ao idoso frágil ou em risco de fragilização.

Os resultados deste trabalho somam-se aos de outros estudos nacionais que discutem o desenvolvimento dos atributos da APS na assistência à população idosa. Acrescenta, na medida em que utiliza amostra representativa da população brasileira mais velha e analisa tal questão à luz dos estratos de fragilidade, ótica que considera a heterogeneidade existente nesta faixa etária. Em relação às outras fases do ciclo de vida, há poucos estudos nesta temática utilizando tal construto. Um trabalho realizado na Região Metropolitana de Belo Horizonte (Minas Gerais) evidenciou que os idosos participantes, de forma geral, avaliaram negativamente os atributos de coordenação do cuidado, orientação familiar e comunitária 15. Em outro estudo, realizado entre idosos residentes em um município da Região Metropolitana de Natal (Rio Grande do Norte), os mesmos identificaram mais problemas nos atributos de orientação familiar, integralidade e acesso. A longitudinalidade, por outro lado, foi o atributo mais bem avaliado 16 . Esses estudos, entretanto, não usaram o conceito de fragilidade, sendo a heterogeneidade dos idosos representada por fatores demográficos e etários. Trabalhos envolvendo diferentes sistemas de saúde como o canadense ${ }^{13}$ e o de Hong Kong 14 também problematizam aspectos relacionados ao acesso, integralidade e longitudinalidade na atenção a idosos e discutem oportunidades de melhorias nas respectivas APS. Tratam, ainda, da presença de barreiras estruturais para a assistência a essa população, como o espaço físico das unidades de saúde, a sinalização e dificuldades com o transporte público, problemas muito frequentes também na realidade brasileira. Embora esses estudos tenham utilizado outro método para discutir o desenvolvimento dos atributos, somente o primeiro o fez na perspectiva da fragilidade.

O construto da fragilidade é uma importante ferramenta tanto para o plano de cuidado individual quanto para a saúde pública 10,27. Estudos têm se dedicado muito à apresentação de instrumentos, formas de definição e classificação da fragilidade, assim como à carga para os sistemas de saúde. Entretanto, há certa lacuna na literatura em relação à qualidade da assistência prestada ou como os sistemas de saúde têm se organizado para prestar adequado atendimento a idosos frágeis e pré-frágeis. Desse modo, a relevância do presente trabalho está na discussão da necessidade de qualificar e reduzir iniquidades na atenção aos idosos frágeis pelos sistemas de saúde, particularmente no que se refere ao maior desenvolvimento dos atributos essenciais da APS, entendendo que este nível de atenção, na perspectiva do envelhecimento populacional, ocupa um papel de destaque para tal assistência. Hipóteses em relação à existência de problemas nessa temática podem ser geradas e mais estudos são necessários para uma avaliação mais pormenorizada.

Vantagens do presente trabalho envolvem a utilização de amostra representativa da população brasileira com 60 anos ou mais e a utilização de método bem definido para a identificação de fragi- 
lidade, permitindo comparações com outros estudos 19 . Há, entretanto, que se considerar algumas limitações, a começar pelo desenho transversal, que permite avaliar associações, mas não a relação temporal entre as variáveis. Outra possível limitação envolve o método utilizado para indicar a presença de problemas nos atributos da APS. Embora não constituam um instrumento validado para tal fim, as questões utilizadas são semelhantes à parte daquelas presentes no instrumento validado mais usado no Brasil 32 e derivam do mesmo marco teórico ${ }^{8}$. Nesse sentido, métodos similares também foram utilizados em outros estudos populacionais 15,33 .

Por fim, apesar da evidente complexidade clínica dos idosos frágeis, a atenção que os serviços de saúde, de forma geral, dedicam a eles pode melhorar. Dentre as várias ações pertinentes, aprofundar no desenvolvimento dos atributos da APS, marcadamente do acesso oportuno, longitudinalidade e integralidade, tem potencial para reduzir iniquidades e qualificar a assistência a essa população, com repercussões favoráveis para a saúde pública e a sustentabilidade de seu sistema.

\section{Colaboradores}

A. M. M. Silva e J. M. Andrade participaram da concepção, planejamento do estudo, análise, interpretação dos dados, redação e revisão crítica do conteúdo do manuscrito. J. V. M. Mambrini, F. B. Andrade e M. F. Lima-Costa colaboraram na coleta de dados, concepção, planejamento do estudo, análise, interpretação dos dados, redação e revisão crítica do conteúdo do manuscrito. Todos os autores aprovaram a versão final.

\section{Informações adicionais}

ORCID: Alexandre Moreira de Melo Silva (00000001-9789-4140); Juliana Vaz de Melo Mambrini (0000-0002-0420-3062); Juliana Mara Andrade (0000-0002-7480-519X); Fabiola Bof de Andrade (0000-0002-3467-3989); Maria Fernanda LimaCosta (0000-0002-3474-2980).

\section{Agradecimentos}

Os autores agradecem a todos os pesquisadores, entrevistadores e participantes do ELSI-Brasil.

\section{Referências}

1. Clegg A, Young J, Iliffe S, Rikkert MO, Rockwood K. Frailty in elderly people. Lancet 2013; 381:752-62.

2. Morley JE, Vellas B, van Kan GA, Anker SD, Bauer JM, Bernabei R, et al. Frailty consensus: a call to action. J Am Med Dir Assoc 2013; 14:392-7.

3. Fried LP, Tangen CM, Walston J, Newman AB, Hirsch C, Gottdiener J, et al. Frailty in older adults: evidence for a phenotype. J Gerontol A Biol Sci Med Sci 2001; 56:M146-56.

4. Faller JW, Pereira DDN, Souza S, Nampo FK, Orlandi FS, Matumoto S. Instruments for the detection of frailty syndrome in older adults: a systematic review. PLoS One 2019; 14:e0216166.

5. Martin FC, Brighton P. Frailty: different tools for different purposes? Age Ageing 2008; 37:129-31

6. Cesari M, Marzetti E, Thiem U, Pérez-Zepeda UM, Van Kan GA, Landi F, et al. The geriatric management of frailty as paradigm of "the end of the disease era". Eur J Intern Med 2016; 31:11-4.

7. Han L, Clegg A, Doran T, Fraser L. The impact of frailty on healthcare resource use: a longitudinal analysis using the Clinical Practice Research Datalink in England. Age Ageing 2019; 48:665-71. 
8. Starfield B. Atenção primária: equilíbrio entre necessidades de saúde, serviços e tecnologia. Brasília: Organização das Nações Unidas para a Educação, a Ciência e a Cultura; Ministério da Saúde; 2002.

9. Vidal EI. Frailty in older adults: perspectives for research and practice in public health. Cad Saúde Pública 2014; 30:1133-5.

10. Ilinca S, Calciolari S. The patterns of health care utilization by elderly Europeans: frailty and its implications for health systems. Health Serv Res 2015; 50:305-20.

11. Macinko J, Mendonça CS. The Family Health Strategy, a strong model of primary health care that delivers results. Saúde Debate 2018; 42(spe 1):18-37.

12. Tasca R, Massuda A, Carvalho WM, Buchweitz C, Harzheim E. Recommendations to strengthen primary health care in Brazil. Rev Panam Salud Pública 2020; 44:e4.

13. Giguere AMC, Farmanova E, Holroyd-Leduc JM, Straus SE, Urquhart R, Carnovale V, et al. Key stakeholders' views on the quality of care and services available to frail seniors in Canada. BMC Geriatr 2018; 18:290.

14. Woo J, Mak B, Yeung F. Age-friendly primary health care: an assessment of current service provision for older adults in Hong Kong. Health Serv Insights 2013; 6:69-77.

15. Augusto DK, Lima-Costa MF, Macinko J, Peixoto SV. Factors associated with the evaluation of quality of primary health care by older adults living in the Metropolitan Region of Belo Horizonte, Minas Gerais, Brazil, 2010. Epidemiol Serv Saúde 2019; 28:e2018128.

16. Araújo LUA, Gama ZAS, Nascimento FLA, Oliveira HFV, Azevedo WM, Almeida Júnior HJB. Evaluation of the quality of primary health care from the perspective of the elderly. Ciênc Saúde Colet 2014; 19:3521-32.

17. Lima-Costa MF, Andrade FB, Souza Jr. PRB, Neri AL, Duarte YAO, Castro-Costa E, et al. The Brazilian Longitudinal Study of Aging (ELSI-Brazil): objectives and design. Am J Epidemiol 2018; 187:1345-53.

18. Andrade FB, Lebrão ML, Santos JLF, Duarte YAO. Relationship between oral health and frailty in community-dwelling elderly individuals in Brazil. J Am Geriatr Soc 2013; 61:80914.

19. Andrade JM, Duarte YAO, Alves LC, Andrade FCD, Souza Jr. PRB, Lima-Costa MF, et al. Frailty profile in Brazilian older adults: ELSI-Brazil. Rev Saúde Pública 2018; 52 Suppl $2: 17 \mathrm{~s}$.

20. Simonsick EM, Maffeo CE, Rogers SK, Skinner EA, Davis D, Guralnik JM, et al. Methodology and feasibility of a home-based examination in disabled older women: the Women's Health and Aging Study. J Gerontol A Biol Sci Med Sci 1997; 52:M264-74.

21. Radloff LS. The CES-D Scale: a self-report depression scale for research in the general population. Appl Psychol Meas 1977; 1:385-401.
22. Fan NM, Lyu J, He P; The IPAQ Group. Guidelines for data processing and analysis of the International Physical Activity Questionnaire (IPAQ) - short and long forms. https://sites. google.com/site/theipaq/scoring-protocol (acessado em 13/Jul/2020).

23. Macklai NS, Spagnoli J, Junod J, SantosEggimann B. Prospective association of the SHARE-operationalized frailty phenotype with adverse health outcomes: evidence from $60+$ community-dwelling Europeans living in 11 countries. BMC Geriatr 2013; 13:3.

24. Andersen RM. Revisiting the behavioral model and access to medical care: does it matter? J Health Soc Behav 1995; 36:1-10.

25. Hoeck S, François G, Geerts J, Van der Heyden J, Vandewoude M, Van Hal G. Health-care and home-care utilization among frail elderly persons in Belgium. Eur J Public Health 2012; 22:671-7.

26. Theou O, Cann L, Blodgett J, Wallace LMK, Brothers TD, Rockwood K. Modifications to the frailty phenotype criteria: systematic review of the current literature and investigation of 262 frailty phenotypes in the Survey of Health, Ageing, and Retirement in Europe. Ageing Res Rev 2015; 21:78-94.

27. Buckinx F, Rolland Y, Reginster JY, Ricour C, Petermans $J$, Bruyère $O$. Burden of frailty in the elderly population: perspectives for a public health challenge. Arch Public Health 2015; 73:19.

28. Moraes EN, Carmo JA, Moraes FL, Azevedo RS, Machado CJ, Montilla DE. Clinical-functional vulnerability index-20 (IVCF-20): rapid recognition of frail older adults. Rev Saúde Pública 2016; 50:81.

29. Heath I, Rubinstein A, Stange KC, van Driel ML. Quality in primary health care: a multidimensional approach to complexity. BMJ 2009; 338:b1242.

30. Wachs LS, Nunes BP, Soares MU, Facchini LA, Thumé E. Prevalência da assistência domiciliar prestada à população idosa brasileira e fatores associados. Cad Saúde Pública 2016; 32:e00048515.

31. Ministério da Saúde. Portaria no 2.528, de 19 de outubro de 2006. Aprova a Política Nacional de Saúde da Pessoa Idosa. Diário Oficial da União 2006; 20 out.

32. Fracolli LA, Gomes MF, Nabão FRZ, Santos MS, Cappellini VK, Almeida ACC. Primary health care assessment tools: a literature review and metasynthesis. Ciênc Saúde Colet 2014; 19:4851-60.

33. Macinko J, Andrade FB, Souza Jr. PRB, LimaCosta MF. Primary care and healthcare utilization among older Brazilians (ELSI-Brazil). Rev Saúde Pública 2018; 52 Suppl 2:6s. 


\section{Abstract}

This study aimed to examine the association between frailty syndrome and the perception of problems in indicators of attributes in primary healthcare (PHC) among elderly Brazilians. This was a cross-sectional study with 5,432 participants 60 years or older in the first wave of the Brazilian Longitudinal Study of Aging (ELSI-Brasil), conducted in 2015 and 2016. Frailty, the independent variable, was defined according to the theoretical framework of the frailty phenotype, and the indicators of problems in PHC attributes, the dependent variables, were obtained from questions related to health services use. Access, longitudinal care, coordination, comprehensiveness, family orientation, and cultural adequacy were the target attributes. For the data analysis, logistic regression models were used, adjusted for predisposing, enabling, and need factors for use of health services. Among the participants, 55.1\% were females, $57.9 \%$ were 60 to 69 years of age, and $51.8 \%$ reported multimorbidity. Frail and pre-frail elders accounted for $13.4 \%$ and $54.5 \%$ of the sample, respectively. Multivariate analyses showed that frail elders (compared to robust elders) showed higher odds of reporting problems with access $(O R=1.45$; 95\%CI: 1.08-1.93), longitudinal care ( $O R=1.54$; 95\%CI: 1.19-2.00), and comprehensive care (OR = 1.45; 95\%CI: 1.14-1.85), in addition to more problems with attributes of $\mathrm{PHC}(\mathrm{OR}=1.38$; 95\% CI: 1.05-1.82, for 5 or more). The study suggests the occurrence of inequities in the care provided by Brazilian PHC for frail elders, particularly in the attributes of access, longitudinal care, and comprehensiveness.

Aging; Frail Elderly; Health Services for Aged; Health Services Research; Primary Health Care

\section{Resumen}

El objetivo de este trabajo fue examinar la asociación entre el síndrome de fragilidad y la percepción de problemas en indicadores de atributos de la atención primaria a la salud (APS) entre ancianos brasileños. Se trata de un estudio transversal implicando a 5.432 participantes, con 60 años o más, del primer grupo del Estudio Longitudinal de la Salud entre Ancianos Brasileños (ELSI-Brasil), realizado entre 2015 y 2016. La fragilidad -variable independiente- fue definida a partir del marco teórico del fenotipo de fragilidad, y los indicadores de problemas en atributos de la APS, variables dependientes, se obtuvieron a partir de preguntas relacionadas con el uso de servicios de salud. Acceso, longitudinalidad, coordinación, integralidad orientación familiar y adecuación cultural fueron los atributos evaluados. Se utilizaron modelos de regresión logística ajustados por factores predisponentes, facilitadores, $y$ de necesidad de uso de servicios de salud para análisis de datos. Entre los participantes, un 55, $1 \%$ eran de sexo femenino, un $57,9 \%$ tenían entre 60 y 69 años y un 51,8\% informaron de multimorbilidad. Los ancianos frágiles y prefrágiles representaron un 13,4\% y un 54,5\% de la muestra, respectivamente. Los resultados del análisis multivariado mostraron que los ancianos frágiles, en comparación con los fuertes, tuvieron más oportunidades de apuntar problemas de acceso $(O R=1,45$; IC95\%: 1,08-1,93), longitudinalidad (OR = 1,54; IC95\%: 1,19-2,00) e integralidad $(\mathrm{OR}=1,45$; IC95\%: 1,14-1,85), además de un mayor número de problemas en atributos de la APS $(O R=1,38$; IC95\%: 1,05-1,82, para 5 o más). El estudio sugiere la ocurrencia de inequidades en la asistencia prestada por la APS brasileña a ancianos frágiles, particularmente en el ámbito de los atributos acceso, longitudinalidad e integralidad.

Envejecimiento; Anciano Frágil; Servicios de Salud para Ancianos; Investigación sobre Servicios de Salud; Atención Primaria de Salud
Recebido em 28/Ago/2020

Versão final reapresentada em 16/Nov/2020 Aprovado em 17/Dez/2020 